\title{
Metalografía a color en aleaciones AI-Si comerciales. Optimización de las técnicas de caracterización microestructural mediante microscopía óptica de reflexión ${ }^{(\bullet)}$
}

\author{
B. Suárez-Peña*, J. Asensio-Lozano** y G. F. Vander-Voort***
}

\begin{abstract}
Resumen
La demanda de aleaciones con calidades optimizadas conlleva la mejora continua de estas calidades, lo que viene suscitando la necesidad de mejora y optimización permanentes de las técnicas de caracterización metalográfica. Las técnicas de ataque tradicionales, utilizadas en la observación microscópica de las aleaciones de aluminio, no permiten un análisis detallado de la evolución microestructural de las fases presentes ni de parte de los mecanismos que rigen la solidificación. En el presente trabajo, se explora el potencial de la metalografía en color aplicada a las estructuras de solidificación de aleaciones $\mathrm{Al}-\mathrm{Si}$ 12. Se ha utilizado un procedimiento de ataque en color, basado en un reactivo desarrollado por Weck para aleaciones de aluminio ${ }^{[1]}$. El empleo de esta técnica ha permitido la evaluación cualitativa de la microestructura. La técnica ha permitido revelar características microestructurales que los métodos de ataque en blanco-negro no revelan.
\end{abstract}

Palabras clave

Metalografía en color; Aleaciones aluminio-silicio; Reactivo de Weck; Microscopía óptica.

\section{Colour metallography in commercial Al-Si alloys. Optimization of the microstructural characterization techniques in light optical microscopy}

\begin{abstract}
The present demand on alloy production with improved quality requires the optimization of the metallographic procedures used on its characterization. Traditional etching techniques commonly employed for phase identification by optical metallography in aluminium alloys are not always suitable for a detailed analysis of existing phases, nor to accurately predict the mechanisms that govern the solidification process in certain detail. This work explores the potential of colour metallography to reveal at its best as-cast microstructures in $\mathrm{Al}-\mathrm{Si} 12$ alloys. For this purpose a colour etching technique, specifically developed for aluminium alloys and based on the Weck reagent ${ }^{[1]}$. The application of such etchant has allowed the qualitative characterization of the microstructure. And it has also shown the advantages of colour metallography over black and white $(B \& W)$ etching techniques.
\end{abstract}

Keywords

Colour metallography; Al-Si alloys; Weck reagent; Optical microscopy.

\section{INTRODUCCIÓN}

Habitualmente, las principales fases presentes en las aleaciones del sistema Al-Si son: granos de solución sólida, Al- $\alpha$; eutéctica laminar, Al-Si, con geometría acicular en el plano de pulido; además, de silicio cuboidal y un compuesto intermetálico de base hierro, muy frágil, que puede presentar varias morfologías: poliédrica, chinese script $(\alpha-\mathrm{AlFeSi})$ y laminar
( $\beta$ - $\mathrm{AlFeSi})$; este ultimo se observa acicular en las micrografías 2D [2].

La presencia de elementos como titanio y estroncio modifica la microestructura de esta aleación. La adición de titanio provoca el aumento de la fracción en volumen y el afino de la fase $\mathrm{Al}-\alpha^{[3]}$. El estroncio promueve, por un lado, cambios en la morfología de la eutéctica, que se transforma en globular ${ }^{[4]} \mathrm{y}$, por otro, induce una disminución en el tamaño de los

\footnotetext{
(•) Trabajo recibido el día 2 de Marzo de 2010 y aceptado en su forma final el día 3 de Mayo de 2010

* Departamento de Ciencia de los Materiales e Ingeniería Metalúrgica, E.U.I.T.I. de Gijón, Campus de Biseques, Universidad de Oviedo 33203, Gijón (Asturias), ESPAÑA.

** Departamento de Ciencia de los Materiales e Ingeniería Metalúrgica, E.T.S.I.M.O., C/ Independencia,13, Universidad de Oviedo, 33004,

Oviedo (Asturias), ESPAÑA. jasensio@uniovi.es.

*** Senior Research Consultant, Metallography Specialist, Lake Bluff, Illinois (US).
} 
cuboides de silicio y un aumento en la fracción en volumen de la fase $\mathrm{Al}-\alpha^{[5]}$. Estos cambios mejoran sustancialmente las propiedades mecánicas de las piezas fabricadas a partir de estas aleaciones: se observan aumentos en la resistencia mecánica, la ductilidad, la tenacidad, etc. Todo ello, ha llevado a los investigadores a tratar de mejorar su microestructura ${ }^{[6]}$, en particular, la de la aleación eutéctica, ampliamente utilizada en la industria de automoción.

La optimización de la calidad de las aleaciones, implica la necesidad de disponer de técnicas de caracterización metalográfica que permitan determinar las principales características microestructurales: fases, bordes de grano, precipitados, etc. Los métodos metalográficos establecen instrucciones precisas sobre la preparación y ataque de las muestras ${ }^{[7]}$, aunque la complejidad de algunas técnicas las hace escasamente operativas. Además, muchos métodos que son efectivos a la hora de determinar ciertas fases en aleaciones comerciales, sin embargo, no sirven o producen resultados diferentes al introducir modificaciones en ellas ${ }^{[8]}$.

La investigación de nuevos procedimientos o la aplicación de los ya existentes a aleaciones diferentes permiten descubrir nuevos caminos. Tal es el caso de la metalografía en color. Se trata de una técnica que, tras el ataque, crea un film superficial que provoca un efecto de color en reflexión. Los reactivos de ataque utilizados en esta técnica pueden colorear las fases anódicas y las catódicas. Estas últimas suelen teñirse de un solo color, debido a que el film que se forma sobre ellas, habitualmente, tiene un espesor uniforme. La coloración de las fases anódicas depende del espesor del film creado. Una vez formado el film, la velocidad de ataque desciende. A medida que el film se engruesa, se producen colores visibles a través del microscopio óptico, secuenciados como sigue:

\section{Amarillo $\rightarrow$ Rojo $\rightarrow$ Violeta $\rightarrow$ Azul $\rightarrow$ Verde}

Estas tonalidades son función, por un lado, de la tendencia del sustrato a verse atacado por el reactivo: las áreas más anódicas experimentan la secuencia más rápidamente; y, por otro, del tiempo de inmersión: la elección del tiempo de ataque es crítica a la hora de crear un contraste adecuado entre las fases ${ }^{[9}$ y 10$]$.
La técnica más sencilla de ataque coloreado es el ataque por inmersión. Su uso comenzó a extenderse a raíz del desarrollo de reactivos por investigadores como Klemm ${ }^{[11 \text { y 12] y Beraha }}{ }^{[13-17]}$. Posteriormente, fueron desarrollados por Benscoter, Kilpatrick

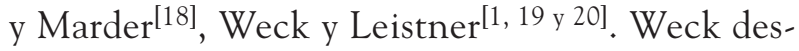
arrolló numerosos reactivos de ataque para metalografía en color de aleaciones de titanio y aluminio, que permitieron revelar al microscopio óptico características microestructurales, tales como la segregación dendrítica en el aluminio, los precipitados intermetálicos en la región interdendrítica, en aleaciones $\mathrm{AlCuMg}$, la estructura granular, en las aleaciones para forja AA 6061-T6 o la intercara entre el metal de base y la soldadura, en aleaciones de $\mathrm{AlCuMn}^{[21]}$.

En los últimos años, algunos investigadores han conducido sus estudios sobre aleaciones de aluminio utilizando la metalografía en color como herramienta de análisis ${ }^{[9]}$. Sin embargo, en la mayoría de los casos, los colores desarrollados tras el empleo de esta técnica han sido escasamente interpretados. El análisis de la visualización en microscopía óptica y su correlación con la evolución microestructural de las aleaciones de aluminio no ha sido resuelto, aún.

En el presente trabajo se ha intentado probar técnicas de ataque y observación microscópica que posibiliten mejoras en el contraste entre fases y simplifiquen la caracterización metalográfica de las aleaciones Al-Si eutécticas.

La herramienta utilizada para la caracterización de estas aleaciones ha sido la metalografía a color. Se ha analizado la evolución de las principales fases presentes durante su proceso de solidificación. El reactivo de ataque empleado ha sido una solución básica de permanganato potásico desarrollada por Weck. Tras su empleo, se forma un film superficial que crea contrastes de color, revelando información que no puede obtenerse con los reactivos de ataque en blanco-negro, convencionales, a base de soluciones acuosas con diferentes concentraciones de HF. Su uso ha permitido revelar, mediante microscopía óptica, características microestructurales difíciles de observar en este tipo de aleaciones.

Tabla I. Composición química de la aleación comercial Al-Si 12 (\% en peso)

Table I. Chemical composition of Al-12 \% Si commercial alloy (percentage in weight)

\begin{tabular}{cccccccccccc}
\hline $\mathrm{Si}$ & $\mathrm{Fe}$ & $\mathrm{Cu}$ & $\mathrm{Mn}$ & $\mathbf{M g}$ & $\mathrm{Ni}$ & $\mathrm{Zn}$ & $\mathrm{Pb}$ & $\mathrm{Ti}$ & $\mathrm{Cr}$ & Other & $\mathrm{Al}$ \\
\hline 12,90 & 0,82 & 0,04 & 0,20 & 0,01 & 0,006 & 0,03 & 0,02 & 0,008 & 0,013 & 0,023 & bal. \\
\hline
\end{tabular}


METALOGRAFIAA A COLOR EN ALEACIONES AL-SI COMERCIALES. OPTIMIZACIÓN DE LAS TÉCNICAS DE CARACTERIZACIÓN MICROESTRUCTURAL MEDIANTE MICROSCOPIA ÓPTICA DE REFLEXIÓN COLOUR METALLOGRAPHY IN COMMERCIAL AL-SI ALLOYS. OPTIMIZATION OF THE MICROSTRUCTURAL CHARACTERIZATION TECHNIQUES IN LIGHT OPTICAL MICROSCOPY

\section{PROCEDIMIENTO EXPERIMENTAL}

La composición de la aleación Al-Si caracterizada se encuentra recogida en la tabla I. Tras seleccionar un lingote se extrajeron muestras metalográficas. En la operación de corte se utilizaron discos de $\mathrm{SiC}$ y diamante. Posteriormente fueron montadas sobre una resina y se desbastaron hasta conseguir su total planitud. El desbaste se llevó a cabo con agua y discos abrasivos de $\mathrm{SiC}$ de granulometría creciente: 220 , 320, 500,800 y 1.200 . El pulido se ejecutó en dos etapas: en la fase inicial se utilizó como lubricante aceite con paños de pulido de seda y poliéster. El abrasivo aplicado fue pasta de diamante de 9,3 y $1 \mathrm{~mm}$, sucesivamente, empleándose tiempos de 5, 5 y 3 min, respectivamente, en cada etapa. El proceso se completó con un pulido de 2-3 min de duración, con paños de gamuza y gel de sílice.

$\mathrm{El}$ posterior ataque de las muestras se realizó a temperatura ambiente utilizando, en unos casos, solución acuosa de $\mathrm{HF}$ al 0,5 \%, y, en otros, el reactivo desarrollado por Weck para aleaciones de aluminio. Este último consiste en una solución de permanganato potásico, hidróxido sódico y agua en las proporciones siguientes:

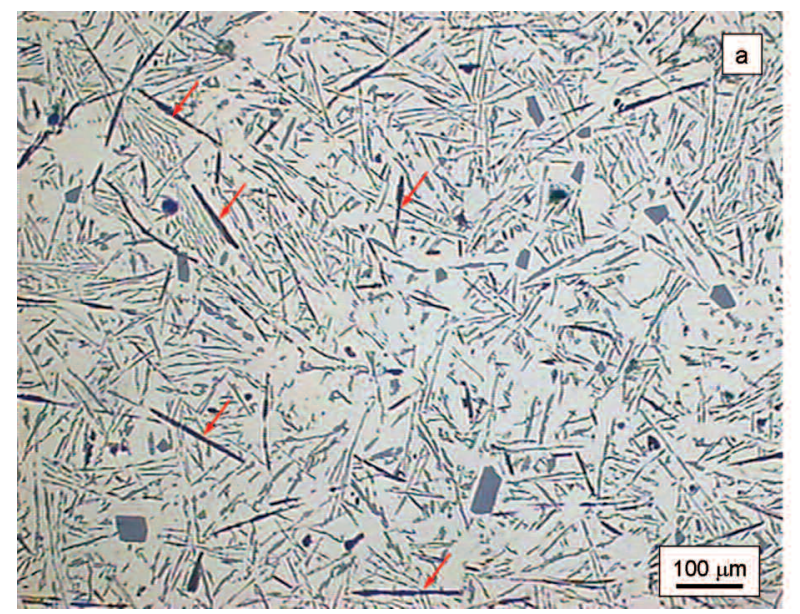

- Agua destilada (100 ml)

- $\mathrm{KMnO}_{4}(4 \mathrm{~g})$

$-\mathrm{NaOH}(1 \mathrm{~g})$

Se sumergieron las muestras pulidas en la solución de ataque, agitando ligeramente hasta obtener el contraste visual. En algunos casos, fueron necesarios varios ciclos de pulido y ataque, hasta obtener el adecuado contraste de los microconstituyentes. En la siguiente sección del presente trabajo, se describirán detalles más precisos sobre el proceso de ataque químico aplicado a las muestras.

El examen de las muestras se llevó a cabo mediante microscopía óptica, utilizando iluminación en campo claro. En otras ocasiones, la anisotropía de las fases observadas posibilitó el uso de la luz polarizada.

\section{RESULTADOS Y DISCUSIÓN}

Un examen inicial de la aleación Al-Si 12, tras el ataque, durante aproximadamente $10 \mathrm{~s}$, con un reactivo en blanco-negro tradicional ( $\mathrm{HF}$ al 0,5\%) revela las principales fases presentes, como reaprecia en la figura $1 \mathrm{a}$ ): dendritos de $\mathrm{Al}-\alpha$ primarios, silicio

Figura 1. Microestructura de la aleación Al-Si 12 atacada con una solución de ácido fluorhídrico al $0,5 \%$ en volumen: (a) Se observan dendritos de Al- $\alpha$ primarios, silicio cuboidal, la fase eutéctica y los compuestos intermetálicos AIFeSi señalados con flechas sobre la micrografía, estos dos últimos con morfología acicular; (b) a mayores aumentos resulta imposible distinguir la interfase entre el aluminio de la eutéctica y el de los dendritos primarios. Los cuboides de silicio primario se indican con flechas.

Figure 1. Microstructure of Al-Si 12 alloy after etching with $0.5 \%$-vol.HF aqueous solution: (a) $\alpha$-Al primary dendrites, Si-cuboids, eutectic phase and the intermetallic compounds of AlFeSi marked with arrows in the micrograph. The last two features exhibiting an acicular mophology in the plane of sectioning; (b) at higher magnification it is indistinguisheable the interphase between the aluminium in the eutectic and that in the dendrites. Primary dendrites are pointed out with arrows. 
cuboidal, además de una eutéctica Al-Si y el compuesto intermetálico de $\mathrm{AlFeSi}$, ambos, con morfología acicular. A mayores aumentos, la micrografía b) amplía una zona de la micrografía a), haciendo patentes la imposibilidad de distinguir la interfase entre el aluminio de la eutéctica y el de los dendritos primarios. La existencia de dendritos de Al- $\alpha$ primarios y silicio cuboidal indica que las condiciones de solidificación no son de equilibrio y refleja la complejidad de dicho proceso, en aleaciones industriales con distintos grados de pureza en los fundidos.

La morfología de los dendritos, tras la solidificación, está vinculada a la cantidad de elementos de aleación presentes en la muestra. En su formación, está latente la tendencia del sistema a minimizar el subenfriamiento constitucional, originado a partir del soluto segregado en la intercara de solidificación. El espaciado entre los brazos dendríticos secundarios (SDAS, o secondary dendrite arm spacing) es mayor en aquellas aleaciones con elevadas concentraciones de elementos solutos con bajo coeficiente de reparto en el aluminio. Dicha segregación de soluto en el líquido, incrementa el subenfriamiento constitucional, lo que supone un estado de desequilibrio que el sistema tiende a restaurar. Con este propósito, los mecanismos que ocurren simultáneamente son:

- Por un lado, la homogeneización del líquido enriquecido en soluto, mediante procesos difusionales en el mismo.

- Y, por otro, un aumento de la intercara S/L, que persigue distribuir en un mayor volumen de líquido, el soluto que el sólido rechaza en su crecimiento. De esta forma, las estructuras dendríticas desarrolladas resultarán ramificadas y presentarán un aumento del SDAS [22].

La medida de este parámetro microestructural en las aleaciones para moldeo resulta de interés, no sólo a la hora de resolver la ecuación de Hall-Petch para la determinación de las propiedades mecánicas, en concreto, el límite elástico, sino, también, cuando se intenta estimar el tiempo de solidificación local en las simulaciones de los procesos de moldeo ${ }^{[23}$ y 24]. En la figura 1, puede observarse que los granos de Al- $\alpha$ están contorneados por la eutéctica interdrendrítica, aunque el contraste entre ambas fases es escaso y la interfase no se aprecia como una línea continua. En definitiva, ni la definición ni el contraste entre las fases son los adecuados, si se pretenden detallar las características microestructurales.

El componente mayoritario presente en la aleación, el aluminio, es un metal muy activo, como se deduce de su posición en la serie electroquímica de potenciales normales de electrodo, pero se pasiva por exposición al aire ó al agua. Godard y sus colaboradores ${ }^{[25]}$ sugirieron que el producto inicial de la corrosión del aluminio en un ambiente acuoso es el hidróxido de aluminio:

$$
2 \mathrm{Al}+6 \mathrm{H}_{2} \mathrm{O} \rightarrow 2 \mathrm{Al}(\mathrm{OH})_{3}+3 \mathrm{H}_{2}
$$

Posteriormente, se originará un oxido hidratado $\left(\mathrm{Al}_{2} \mathrm{O}_{3} \cdot \mathrm{H}_{2} \mathrm{O}\right)$.

Al sumergir las muestras en el reactivo de ataque para su observación microestructural se produce un fenómeno de corrosión controlado. El aluminio resiste la corrosión, en medio neutro, pero se corroe con rapidez, en medio alcalino. Según el diagrama de Pourbaix para el aluminio[26], la capa de óxido superficial formada según la reacción (1), es estable a temperatura ambiente para valores de $\mathrm{pH}$ entre 4 y 8,5 . Sin embargo. cuando se sumerge la muestra para su ataque en el reactivo desarrollado por Weck, basado en una solución alcalina de permanganato potásico, el $\mathrm{pH}$ alcanza un valor, aproximadamente, de 13, por encima del indicado en el rango anterior. Por tanto, bajo la acción del hidróxido sódico, la capa de oxido formada por corrosión se disuelve, dejando al descubierto el aluminio metálico:

$$
\begin{gathered}
\mathrm{Al}(\mathrm{OH})_{3}+\mathrm{NaOH} \rightarrow \mathrm{Na}^{+}+\mathrm{Al}(\mathrm{OH})_{4}^{-} \\
\mathrm{Al}_{2} \mathrm{O}_{3}+2 \mathrm{NaOH}+3 \mathrm{H}_{2} \mathrm{O} \rightarrow 2 \mathrm{Na}^{+}+\mathrm{Al}(\mathrm{OH})_{4}^{-}
\end{gathered}
$$

Kulinich y sus colaboradores ${ }^{[27]}$ apuntan, en sus trabajos de investigación, que las semi-reacciones tras la disolución de la capa de óxido podrían ser:

$$
\begin{gathered}
\mathrm{Al}+4 \mathrm{OH}^{-} \rightarrow \mathrm{Al}(\mathrm{OH})_{4}^{-}+3 \mathrm{e}^{-} \\
\mathrm{MnO}_{4}^{-}+2 \mathrm{H}_{2} \mathrm{O}+3 \mathrm{e}^{-} \rightarrow \mathrm{MnO}_{2}+4 \mathrm{OH}^{-}
\end{gathered}
$$

En definitiva:

$$
\mathrm{Al}+\mathrm{MnO}_{4}^{-}+2 \mathrm{H}_{2} \mathrm{O} \rightarrow \mathrm{Al}(\mathrm{OH})_{4}^{-}+\mathrm{MnO}_{2}
$$

Como producto de esta reacción controlada en medio básico, se genera un film coloreado.

Durante la solidificación de la aleación objeto de estudio, tienen lugar fenómenos de segregación en los dendritos de Al- $\alpha$ generándose diferencias entre los potenciales electroquímicos de los bordes y del interior de los granos. Además, el aluminio de la matriz eutéctica presentará un comportamiento anódi-

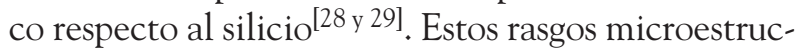
turales se ponen de manifiesto bajo la acción del reactivo de ataque y pueden observarse a través del microscopio óptico. Así, las figuras 2 a) y b), reve- 
METALOGRAFIAA A COLOR EN ALEACIONES AL-SI COMERCIALES. OPTIMIZACIÓN DE LAS TÉCNICAS DE CARACTERIZACIÓN MICROESTRUCTURAL MEDIANTE MICROSCOPIA ÓPTICA DE REFLEXIÓN COLOUR METALLOGRAPHY IN COMMERCIALAL-SI ALLOYS. OPTIMIZATION OF THE MICROSTRUCTURAL CHARACTERIZATION TECHNIQUES IN LIGHT OPTICAL MICROSCOPY

lan la microestructura de la aleación Al-Si 12 tras el ataque con el reactivo desarrollado por Weck. Se han empleado tiempos de ataque, en torno a $20 \mathrm{~s}$. La observación, a través del microscopio óptico utilizando iluminación en campo claro, muestra algunos dendritos de fase $\mathrm{Al}-\alpha$ y secciones de brazos dendríticos secundarios. Tras el ataque, es posible apreciar, por un lado, la regularidad de los brazos dendríticos y su crecimiento en direcciones definidas y, por otro, que en la matriz eutéctica, el silicio acicular no resulta atacado, presentando la tonalidad gris propia de la observación en muestra pulida sin ataque.

Empleando tiempos de ataque superiores, en el entorno de los 30 s y utilizando el mismo reactivo de Weck, la observación con iluminación en campo claro permite apreciar la intercara entre los dendritos de Al- $\alpha$ y la eutéctica Al-Si, como una línea continua (Fig. 2 c) y d)); las diferentes tonalidades de los
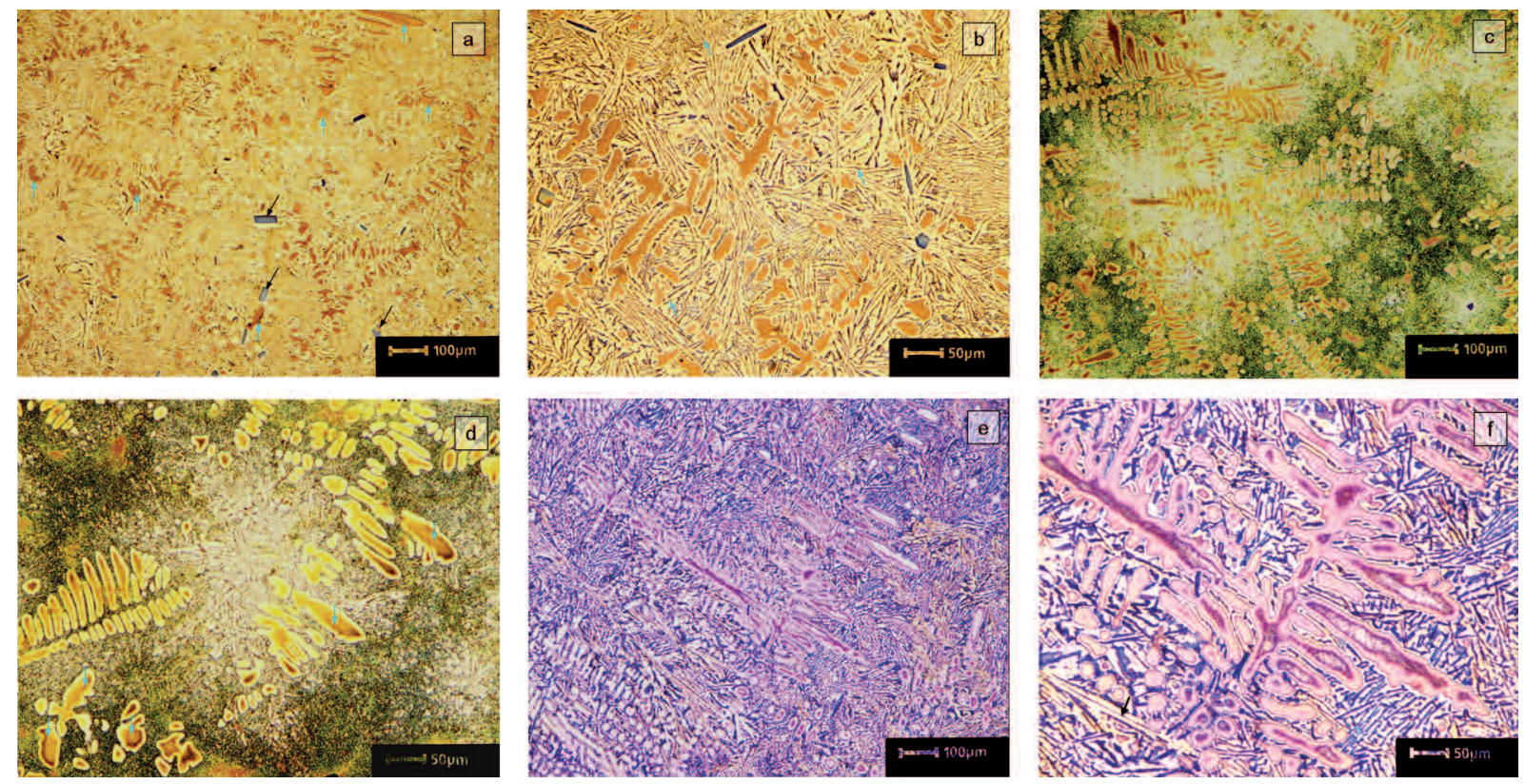

Figura 2. Microestructura de la aleación Al-Si 12 atacada con una solución básica de permanganato potásico sugerida por Weck ${ }^{[1]}$. La observación microscópica en campo claro permite apreciar: (a) dendritos de fase Al- $\alpha$, señalados con flechas verticales en azul claro y secciones de brazos dendríticos secundarios. Los cuboides de silicio aparecen marcados con flechas oblicuas oscuras; y (b) en la matriz eutéctica el Si acicular no resulta atacado, presentando la tonalidad gris propia del estado pulido, y se señala con flechas sobre la propia micrografía. Empleando tiempos de ataque superiores se distingue: (c) la intercara entre los dendritos de Al$\alpha$ y la eutéctica Al-Si se muestra como una línea continua; y (d) las diferencias en las coloraciones evidencian la microsegregación de los elementos en solución de la aleación, (los dendritos están marcados por flechas). Con el empleo de luz polarizada y un filtro de color: (e) se aprecian áreas amarillentas con agujas moradas de compuestos intermetálicos de base Fe desarrollados en la eutéctica; y (f) se constata que la eutéctica no presenta segregación, y en esta última se señala con una flecha una aguja de AlFeSi-acicular.

Figure 2. Al-Si 12 microstructure after etching with an alcaline solution of potasium permanganate (after Weck ${ }^{[1]}$ ). The observation under bright field illumination shows: (a) $\alpha$-Al dendrites pointed out with light blue vertical arrows on the micrograph, and its secondary dendrite arm sections. Cuboidal silicon have been marked with oblique dark arrows; (b )in the eutectic matrix, acicular silicon remains unetched, show a grey colour typical of the unetched condition and it has been underlined with arrows on the micrograph. Under somewhat longer etching times, it is possible to distinguish: (c) the $\alpha-A /$ dendrite and the eutectic interphase is revealed as a continuous line; and (d) the differences observed in colour evidence the microsegregation of solute elements (dendrites are pointed out with arrows). Under polarized light and a colour filter it is possible to distinguish: (e) yellow tinted areas in presence with purple needles of intermetallic AlFeSi compounds; and (f) non-segregated eutectic, acicular AlFeSi being marked with an arrow. 
microconstituyentes permiten diferenciar ambas fases.

Los dendritos, se tiñen de un tono marrón-rojizo, aunque la intensidad de color decrece, gradualmente, hacia los bordes, que muestran una tonalidad amarilla. Las diferencias en las coloraciones evidencian la microsegregación de los elementos en solución de la aleación durante el proceso de solidificación. Estas migraciones, en dirección al frente de solidificación, le confieren un carácter catódico al contorno de los dendritos. Recíprocamente, la mayor pureza en aluminio del núcleo dendrítico, le otorga un carácter anódico y propicia su coloración activa. El ataque revela el comportamiento anódico de la matriz eutéctica. En términos generales, está sobreatacada y adopta una coloración verdosa. Sin embargo, es posible apreciar regiones de eutéctica exentas de coloración. Una posible explicación puede obtenerse a partir de la observación, en detalle, de las mismas. En efecto, cuando el área de eutéctica es pequeña y se encuentra confinada entre colonias de dendritos de $\mathrm{Al}-\alpha$, el carácter anódico de la matriz resulta atenuado por la preponderancia local del carácter catódico del dispersoide: el resultado es una menor tendencia al ataque, de estas colonias de eutéctica, Al-Si.

Es preciso pasar a la observación de la muestra bajo luz polarizada y un filtro de color, tal comno se ilustra en la figura 2 e) y f), para poder discernir los constituyentes en la eutéctica. Así, desaparecen las referencias anódicas y catódicas y la observación permite diferenciar, en la matriz, el silicio, de color azulvioleta y el aluminio, con coloración blanca. El corazón de los dendritos, la zona más rica en aluminio, aparece teñido de color rojizo. Se aprecian áreas amarillentas con agujas moradas indicativas de fases de hierro presentes en la eutéctica. El empleo de la luz polarizada en el estudio de la muestra optimiza los resultados para una adecuada caracterización metalográfica, siempre y cuando las fases objeto de análisis presenten anisotropía óptica ${ }^{[30 \text { y } 31]}$. La eutéctica binaria del sistema Al-Si, debido a la naturaleza de la transformación que la origina, no presenta microsegregación. En este sentido, las técnicas de microscopía óptica en blanco y negro no son aptas para la observación del agregado eutéctico (Fig.1). Por ello, la observación del agregado eutéctico mediante metalografía en color con luz polarizada, permite constatar la ausencia de dicha microsegregación, según se evidencia en la figura 2 e) y f).

Suele ser habitual, en este tipo de aleaciones, la presencia de elementos afinantes (titanio) y modificadores (estroncio). En la figura 3, se muestran las micrografías correspondientes a la aleación comercial Al-Si 12 objeto de estudio, afinada con un $0,03 \%$ de titanio y modificada con $0,05 \%$ de es-
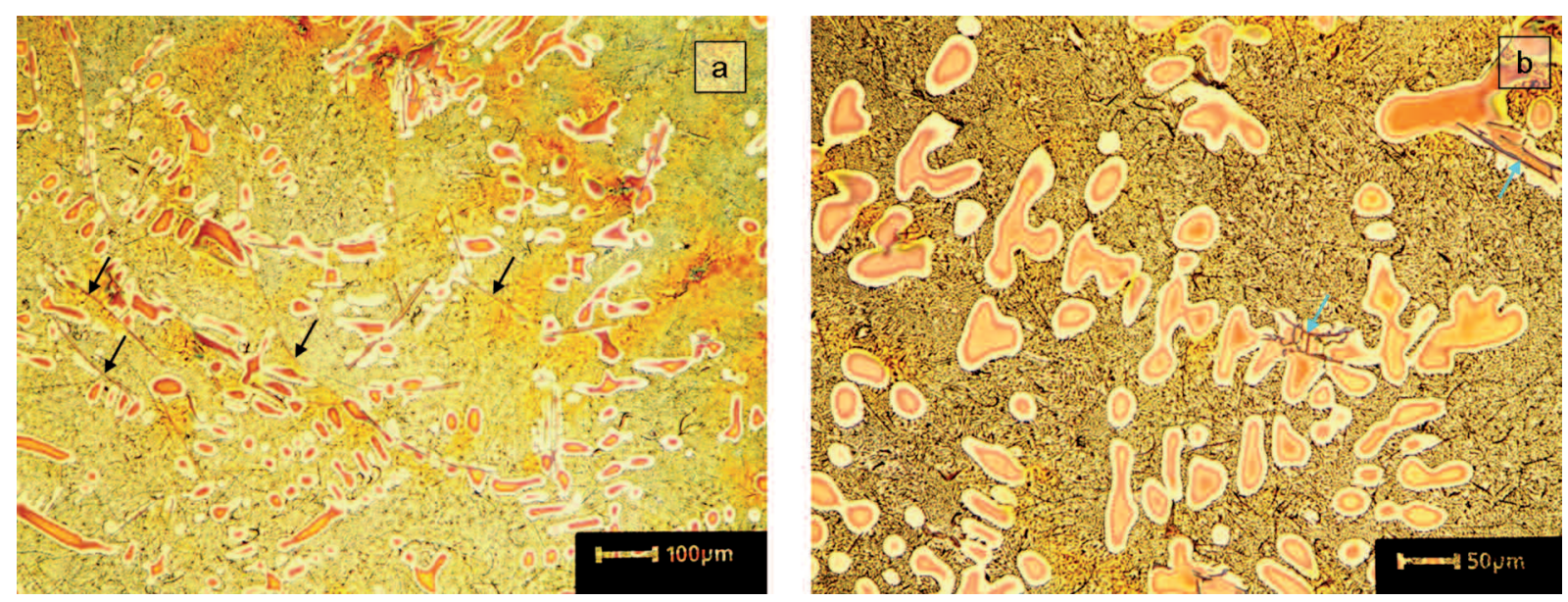

Figura 3. La observación microscópica en campo claro, tras el ataque con el reactivo de Weck, de la aleación Al-Si 12 afinada con un 0,03 \% de Ti y modificada con 0,05 \% de Sr revela: (a) una fase Al- $\alpha$ primario de tipo mixto (columnar-celular) y una eutéctica de gran finura (granular), y se señalan con flechas las agujas de $\beta$-AlFeSi; y (b) a mayores aumentos se observa la existencia del intermetálico $\alpha$-AIFeSi ("chinese script"), marcado con flechas en la micrografía.

Figure 3. Bright field observation after etching with Weck's reagent on a Al-Si 12 refined with $0.03 \%$-wt Ti and modified with 0.05\%-wt Sr: (a) primary $\alpha$-Al with a columnar-cellular mixed structure embedded in a fine and granular eutectic and $\beta$-AlFeSi acicules are pointed out with acicules; (b) at higher magnification it is possible to distinguish another intermetallic: $\alpha$-AlFeSi ("chinese script") also marked with arrows on the micrograph. 
troncio. Los detalles de la adición del afinante y del modificador se encuentran consignados en trabajos de investigación previos de los autores ${ }^{[32]}$. La observación, tras el ataque con el reactivo de Weck, mediante microscopía óptica e iluminación en campo claro, revela una fase Al- $\alpha$ de tipo mixto columnar-celular y una eutéctica de gran finura. Este cambio en la morfología permite apreciar la intercara entre ambas fases como una línea continua. En coincidencia con los resultados anteriores, las tonalidades adoptadas por los microconstituyentes constatan la existencia de segregación dendrítica. En la figura 3 b), a mayores aumentos, se observan, además, dos tipos de compuestos intermetálicos $\alpha$-AlFeSi y $\beta$-AlFeSi. Los primeros, con morfología de caracteres de la escritura china ó chinese scripts, crecen esporádicamente y sirven como nucleantes de la fase $\mathrm{Al}-\alpha$, denotando su naturaleza pro-eutéctica, mientras que los segundos son laminares, aunque se presentan como aciculares en el plano de pulido y aparecen mezclados con la eutéctica, revelando que su nucleación ha tenido lugar conjuntamente con esta.

\section{CONCLUSIONES}

En el presente trabajo, se ha utilizado como reactivo una solución alcalina de permanganato potásico; ésta, ha sido desarrollada por Weck para el ataque metalográfico de las aleaciones de aluminio. Su empleo ha permitido, por un lado, revelar "gráficamente" la estructura y los bordes de los granos tras la solidificación, tanto de la aleación comercial eutéctica del sistema Al-Si, como de esta misma aleación tras adicionarle titanio y estroncio, es decir, afinada y modificada; y, por otro, ha posibilitado una estimación de las áreas dendrítica e interdendrítica, poniendo de manifiesto la segregación provocada por los elementos de aleación y la naturaleza de la reacción eutéctica en este tipo de aleaciones.

Se ha encontrado que, cuando se evalúa la segregación dendrítica y las colonias de fase eutéctica, la observación microscópica empleando luz polarizada, proporciona los mejores resultados.

\section{REFERENCIAS}

[1] E. Weck y E. Leistner, Metallographic Instructions for Colour Etching by Immersion III, D.V.S. Verlag GmbH, Dusseldorf, Alemania, 1986, pp. 3-82.

[2] F. Mondolfo, Aluminium Alloys: Structure and Properties, Butterworth, London, 1976, p. 534.
[3] H. Liao, Y. Sun y G. Sun. Mater. Sci. Eng. A 335 (2002) 62-66.

[4] J. E. Gruzlesky, Microstructure development during metalcasting, AFS Des Plaines, Illinois, 2000, pp 230-234.

[5] H. C. Liao y G. X. Sun, Scr. Mater. 48 (2003) 1.035-1.039.

[6] T. H. Sritharan y J. LI, J. Mater. Process. Technol. 63 (1997) 585-589.

[7] Metallography and Microstructures, ASM Speciality Handbook, ASM Int., 2004, pp. $227-$ 313.

[8] D. San Martin, P.E.J. Rivera- Diaz del Castillo, E. Peekstok y S. Van Der Zwaag, Mater. Char. 58 (2007) 455-460.

[9] A. C. Somasekharan y L. E. Murr. J. Mater. Sci. 41 (2006) 5.365-5.370.

[10] Metallography and Microstructures, ASM Specialty Handbbok, ASM International, pp. 297-298.

[11] H. Klemm, Color Etching of the Fine Structure of Metals with Sodium Thiosulfate, Metalkundliche, Verlag Technik, vol 45, Berlin, 1952.

[12] H. Klemm, Pr. Metallogr. 5 (1968) 163-167.

[13] E. Beraha, J. Iron Steel Inst Jpn. 203 (1965) 454458.

[14] E. Beraha, J. Iron Steel Inst Jpn. 204 (1966) 248252.

[15] E. Beraha, Met. Prog. 90 (1966) 135-140.

[16] E. Beraha, Met. Prog. 90 (1966) 13-17.

[17] E. Beraha, J. Iron Steel Inst Jpn. 205 (1967) 866870.

[18] J. R. Kilpatrick, A.O. Benscoter y A.R. Marder, Met. Prog. (1971) 79-84.

[19] E. Weck y E. Leistner, Metallographic Instructions for Colour Etching by Immersion, Part I: Vol 77,Klemm Colour Etching, D.V.S. Verlag GmbH, Alemania, 1982, pp. 3-60.

[20] E. Weck y E. Leistner, Metallographic Instructions for Colour Etchants by Immersion, Part II: Vol 77/II,Beraha Colour Etchants an their Different Variants, D.V.S. Verlag GmbH, Dusseldorf, Alemania, 1983 pp 3-84.

[21] G.F. Vander Voort, Metallography and Microstructures, ASM Specialty Handbbok, ASM International, pp. 493-562.

[22] J. A. Pero-Sanz, Ciencia e Ingeniería de Materiales, CIE DOSSAT 2000, Madrid, España, 2000, pp. 202-205.

[23] J. E. Gruzlesky, Microstructure development during metalcasting, AFS Des Plaines, Illinois, 2000, pp. 100-114.

[24] J. C. Jacquet y W. Hotz, Cast. Met. 4 (1992) 200-225.

[25] H. P. Godard, W. P. Jepson, M. R. Bothwell y R. L. Kane, The corrosion of light metals, Ed. John 
Wiley and Sons, New York, USA, 1967, pp. 3 198.

[26] M. Pourbaix, Aluminium Atlas of Electrochemical Equilibrium in Aqueous Solutions, Ed. Pergamon, Oxford, UK, 1966, pp. 168-176.

[27] S. A. Kulinich, A. S. Akhtar, K. C. Wong y K. A. R. Mitchell, Thin Solid Films 515 (2007) 8.386-8.392.

[28] Aluminum and aluminum alloys, ASM Specialty Handbbok, ASM International, pp. 579-590.
[29] Corrosion of Aluminum and Aluminum Alloys, ASM Specialty Handbbok, ASM Internacional, pp. 25-45.

[30] G.F. Vander Voort, Metallography and Microstructures, ASM Specialty Handbbok, ASM International, pp. 495-496.

[31] J. Chapon y W. Szkliniarz, Mater. Charact. 46 (2001) 149-154.

[32] J. Asensio-Lozano y B. Suárez-Peña, Scr. Mater. 54 (2006) 943-947. 


\section{FE DE ERRATAS (Vol. 46-5)}

En el trabajo: Metalografía a color en aleaciones Al-Si comerciales. Optimización de las técnicas de caracterización microestructural mediante microscopía óptica de reflexión.

Págs. 473 y 474.- Las figuras que se presentan en esas páginas son a color, tal como se muestran a continuación.
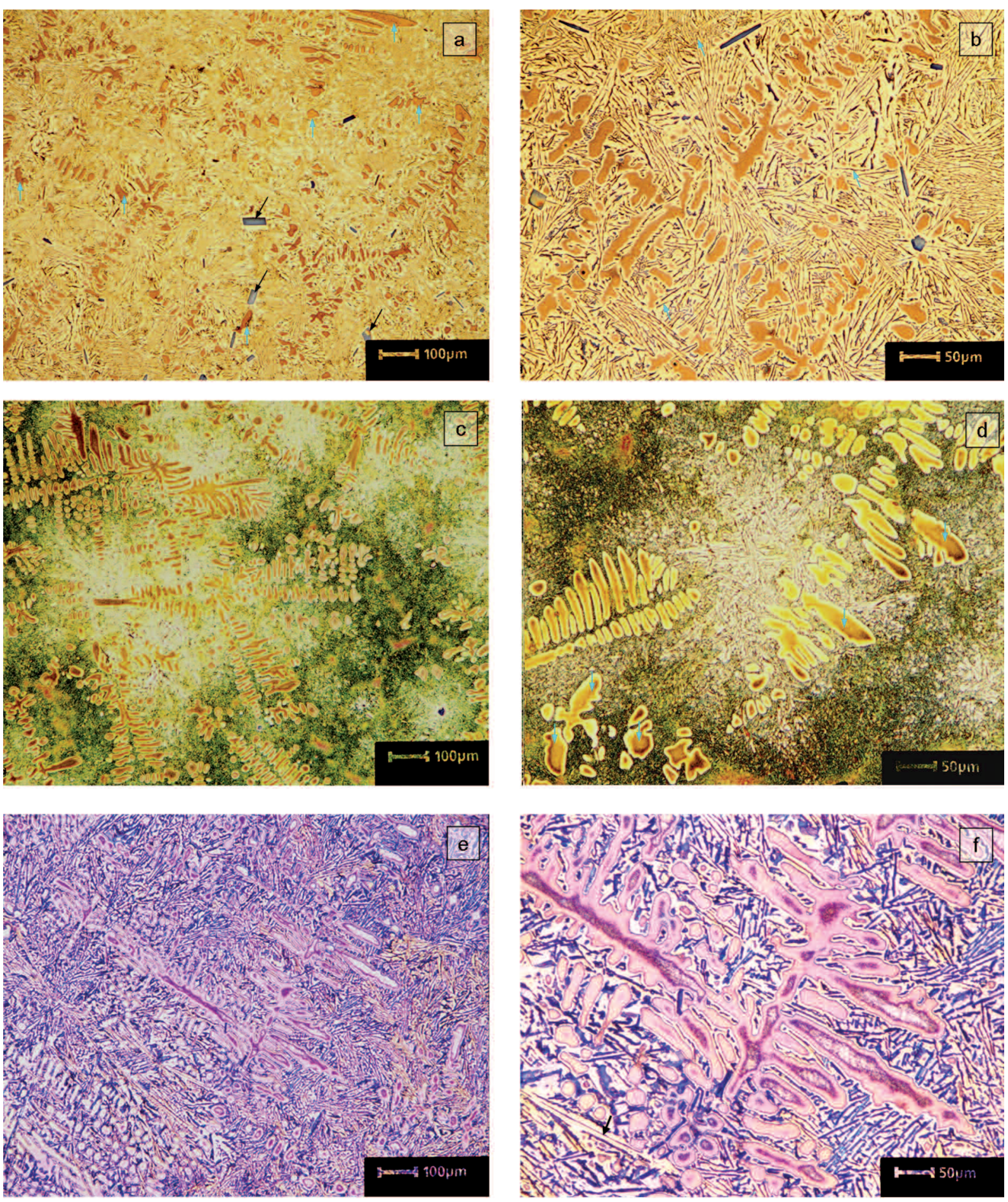

Figura 2. Microestructura de la aleación Al-Si 12 atacada con una solución básica de permanganato potásico sugerida por Weck ${ }^{[1]}$. La observación microscópica en campo claro permite apreciar: (a) dendritos de fase Al- $\alpha$, señalados con flechas verticales en azul claro y secciones de brazos dendríticos secundarios. Los cuboides de silicio aparecen marcados con flechas oblicuas oscuras; y (b) en la matriz eutéctica el Si acicular no resulta 
atacado, presentando la tonalidad gris propia del estado pulido, y se señala con flechas sobre la propia micrografía. Empleando tiempos de ataque superiores se distingue: (c) la intercara entre los dendritos de Al- $\alpha$ y la eutéctica Al-Si se muestra como una línea continua; y (d) las diferencias en las coloraciones evidencian la microsegregación de los elementos en solución de la aleación, (los dendritos están marcados por flechas). Con el empleo de luz polarizada y un filtro de color: (e) se aprecian áreas amarillentas con agujas moradas de compuestos intermetálicos de base Fe desarrollados en la eutéctica; y (f) se constata que la eutéctica no presenta segregación, y en esta última se señala con una flecha una aguja de AlFeSi-acicular.
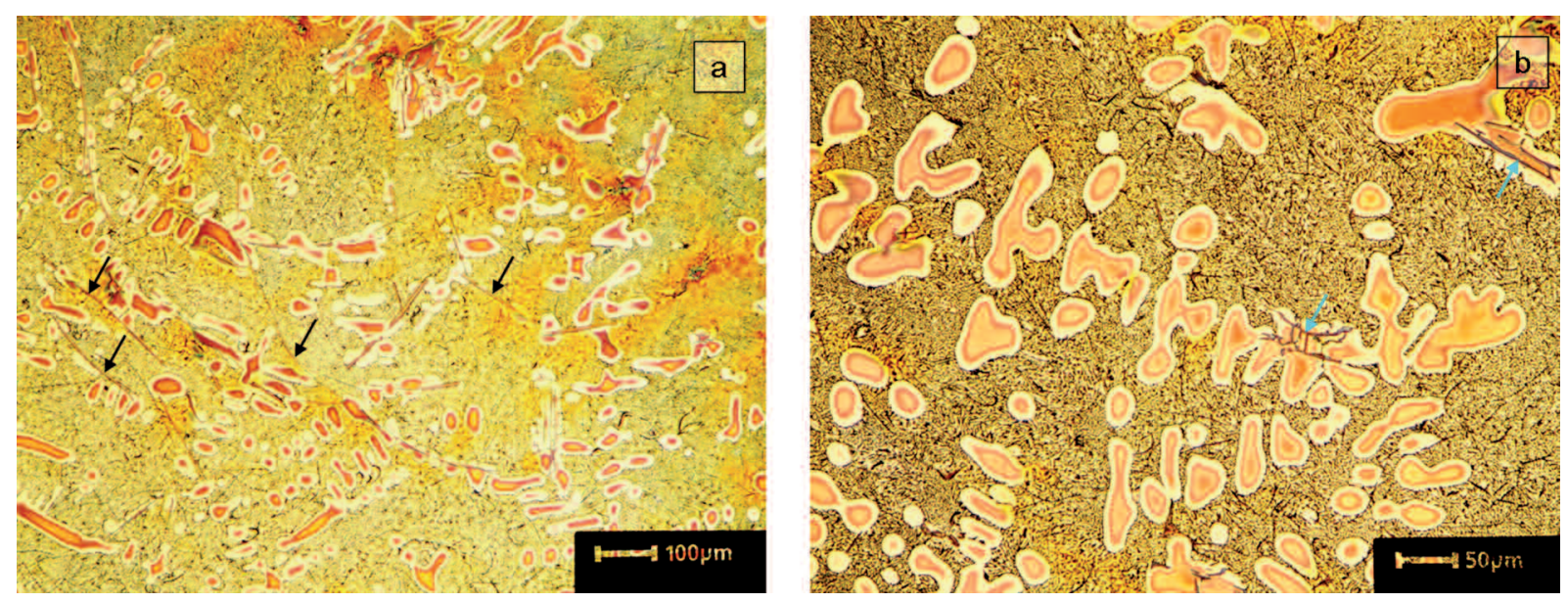

Figura 3. La observación microscópica en campo claro, tras el ataque con el reactivo de Weck, de la aleación Al-Si 12 afinada con un 0,03\% de Ti y modificada con 0,05 \% de Sr revela: (a) una fase Al- $\alpha$ primario de tipo mixto (columnar-celular) y una eutéctica de gran finura (granular), y se señalan con flechas las agujas de $\beta$-AIFeSi; y (b) a mayores aumentos se observa la existencia del intermetálico $\alpha$-AlFeSi ("chinese script"), marcado con flechas en la micrografía. 